\title{
Interleukin 1 receptor antagonist gene polymorphism and restenosis after coronary angioplasty
}

S E Francis, N J Camp, A J Burton, R M Dewberry, J Gunn, A Stephens-Lloyd, D C Cumberland, A Gershlick, D C Crossman

\begin{abstract}
Background-Percutaneous transluminal coronary angioplasty (PTCA) is limited by the recurrence of luminal stenosis, which occurs in up to $50 \%$ of procedures. It has been shown that patient specific factors, perhaps genes, contribute to this process.

Objective-To determine whether completion of healing after PTCA is part of an acute self limiting inflammatory process and whether polymorphism at important inflammatory gene loci might determine susceptibility to restenosis after PTCA.

Design-DNA samples were collected from 171 patients attending for elective PTCA in Sheffield (S) and Leicester (L), who were scheduled to undergo follow up angiography (at four months (L) or six months (S)) as part of other restenosis studies. At follow up angiography, the patients were separated into restenosers $(>50 \%$ luminal narrowing) and non-restenosers $(<50 \%$ luminal narrowing). Four DNA polymorphisms within interleukin 1 (IL-1) related loci (IL-1A (-889), IL-1B (-511), IL-1B (+3954), and IL-1 RN intron 2 VNTR (variable number tandem repeat)) were genotyped using methods based on polymerase chain reaction. Significance was assessed by $\chi^{2}$ analysis of the relevant contingency table, and the magnitude of effect was estimated by calculating odds ratios. The Mantel-Haenszel (MH) test was applied to summarise data across the two populations.

Results-Allele 2 at IL- $1 \mathrm{RN}$ (IL- $1 \mathrm{RN}^{\star} 2$ ) was significantly over represented in the non-restenoser group (L+S, $34 \%$ v $23 \%$ in restenosers). Furthermore, IL-1RN ${ }^{\star} 2$ homozygosity was increased in the non-restenoser population compared with the restenosers (MH test: $\mathrm{p}=0.0196(\mathrm{~L}+\mathrm{S}) ; \mathrm{p}=0.031(\mathrm{~L}+\mathrm{S}$, single vessel disease only), and the effect seemed to be restricted to the single vessel disease subpopulation. For other polymorphism within IL-1 related loci no significant associations were found with either restenosis or non-restenosis.

Conclusions-IL- $1 \mathrm{RN}^{\star} 2$ may be associated with protection from restenosis after PTCA for individuals with single vessel disease. As this polymorphism has functional significance, this finding suggests that alteration in an individual's inflammatory predisposition may modulate the blood vessel response to injury.

(Heart 2001;86:336-340)
\end{abstract}

Cardiovascular

Medicine Group,

Division of Clinical

Sciences, Clinical

Sciences Centre,

University of Sheffield,

Northern General

Hospital, Sheffield

S5 7AU, UK

$S$ E Francis

A J Burton

R M Dewberry

J Gunn

D C Cumberland

D C Crossman

Genetic Research, Intermountain Health

Care and

Epidemiology,

University of Utah,

USA

N J Camp

Division of Cardiology, Glenfield General

Hospital, Leicester, UK

A Stephens-Lloyd

A Gershlick

Correspondence to: Professor Crossman

d.c.crossman@sheffield.ac.uk

Accepted 11 May 2001

Keywords: restenosis; inflammation; angioplasty; interleukin-1 receptor antagonist

Percutaneous transluminal coronary angioplasty (PTCA) is a well established procedure for coronary revascularisation but is limited by the recurrence of luminal stenosis (restenosis), which occurs in up to $50 \%$ of procedures. Early studies suggested a Gaussian distribution of restenosis when it is expressed as a continuous variable (either minimum lumen diameter, per cent stenosis, or late loss). ${ }^{1}$ Recent studies have challenged this view and indicate a bimodal distribution for de novo lesions after PTCA. ${ }^{2}$ It has also been shown that there is within-patient correlation of restenosis, suggesting that patient specific factors, perhaps genes, contribute to the process. ${ }^{3}$ Accordingly, various genetic polymorphisms have been investigated in patients with and without restenosis but no clear data have yet emerged..$^{4-12}$ The study reported here is the first to examine cytokine gene variants.

The inflammatory basis of the arterial wall response to injury is now increasingly recognised as an important pathogenic mechanism. ${ }^{13}$ We have recently established that the inflammatory cytokine interleukin 1 (IL-1) accumulates in the arterial wall after balloon injury. ${ }^{14}$
We have also shown that an informative polymorphism within IL-1 related loci (IL-1 receptor antagonist VNTR (variable number tandem repeat)) determines susceptibility to single vessel coronary artery disease. ${ }^{15}$

As a result of the emerging importance of patient based factors in restenosis, and from our previous data, we speculated that polymorphism within IL-1 loci might determine susceptibility to restenosis after PTCA.

\section{Methods}

STUDY POPULATION

We studied 171 patients who were scheduled to undergo follow up angiography after elective PTCA without stenting as part of other protocols. Leicester (L) and Sheffield (S) were selected owing to the availability of accurate angiographic data on the severity of the stenoses. Quantitative coronary angiography was performed on-line (Philips Integris HM 3000 (S); Siemens Micor (L)). Fifty three patients were electively recruited in Sheffield, where follow up angiography was performed at six months, while 118 patients were recruited 
Table 1 Clinical characteristics of patients with and without restenosis after percutaneous transluminal coronary angioplasty

\begin{tabular}{|c|c|c|c|}
\hline & Restenosis & Non-restenosis & $p$ Value \\
\hline \multicolumn{4}{|l|}{ Leicester } \\
\hline Number of patients & 49 & 69 & \\
\hline Age (years) (mean (SEM)) & $59.1(1.2)$ & $57.1(0.9)$ & NS \\
\hline Women & $12.2(6)$ & $17.4(12)$ & ND \\
\hline Hypertension & $24(12)$ & $17.3(12)$ & ND \\
\hline Smoking & $29(14)$ & $34.7(24)$ & ND \\
\hline Diabetes & $2.0(1)$ & $4.3(3)$ & ND \\
\hline Myocardial infarction & $48.9(24)$ & $43.4(30)$ & ND \\
\hline Multivessel disease (\%) & $48.9(24)$ & $39.1(27)$ & ND \\
\hline \multicolumn{4}{|l|}{ Sheffield } \\
\hline Number of patients & 18 & 35 & \\
\hline Age (years) (mean (SEM)) & $53.6(1.8)$ & $53.9(1.5)$ & NS \\
\hline Women & $17(3)$ & $11.4(4)$ & ND \\
\hline Hypertension & $61.1(11)$ & $37.1(13)$ & ND \\
\hline Smoking & $77.7(14)$ & $74.2(26)$ & ND \\
\hline Diabetes & $5.5(1)$ & $11.4(4)$ & ND \\
\hline Myocardial infarction & $57.1(8)$ & $42.8(15)$ & ND \\
\hline Multivessel disease & 0 & 0 & ND \\
\hline \multicolumn{4}{|l|}{ Sheffield and Leicester } \\
\hline No of patients & 67 & 104 & \\
\hline Age (years) (mean (SEM)) & $58.0(1.4)$ & $55.9(1.0)$ & NS \\
\hline Women & $13.4(9)$ & $15.3(16)$ & NS \\
\hline Hypertension & $34.3(23)$ & $24.0(25)$ & NS \\
\hline Smoking & $41.7(28)$ & $48.0(50)$ & NS \\
\hline Diabetes & $2.98(2)$ & $6.7(7)$ & NS \\
\hline Myocardial infarction & $47.7(32)$ & $43.2(45)$ & NS \\
\hline Multivessel disease & $35.8(24)$ & $25.9(27)$ & NS \\
\hline
\end{tabular}

Values are \% (n) except where stated.

Hypertension is defined as diastolic blood pressure $>95 \mathrm{~mm} \mathrm{Hg}$ (Leicester); systolic blood pressure $>160 \mathrm{~mm} \mathrm{Hg}$.

Smoking: current or former (Sheffield); current (Leicester).

ND, not done; NS, not significant.

\begin{tabular}{|lll|}
\hline Alleles & $11 / 12$ & $2 / 2$ \\
\hline Restenosers & 62 & 3 \\
Non-restenosers & 83 & 17 \\
\hline
\end{tabular}

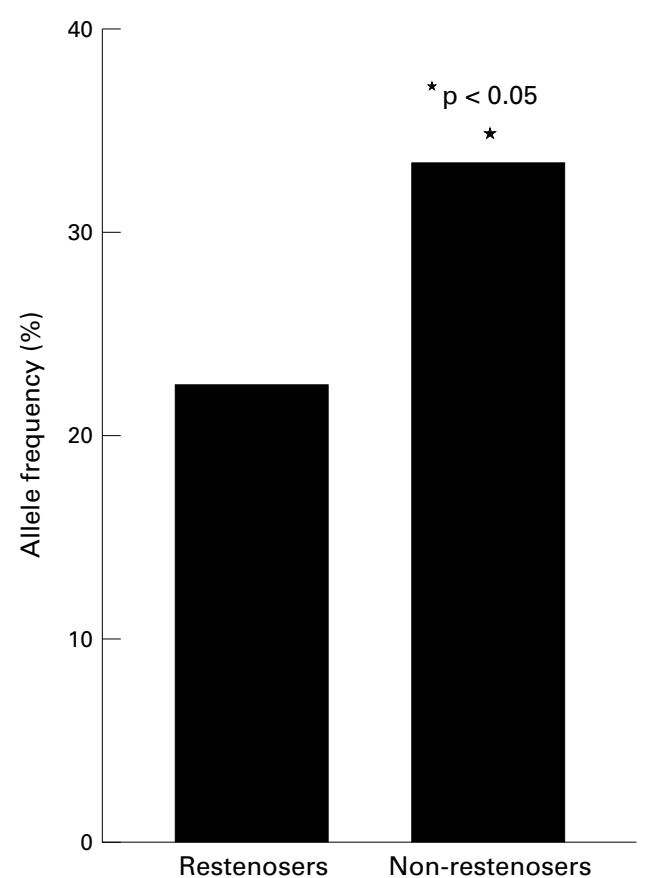

Figure 1 Allele frequency of interleukin 1 receptor antagonist allele $2\left(I L-1 R N^{\star} 2\right)$ in restenosers and non-restenosers (Sheffield $(S)$ and Leicester $(L)$ ). Text box indicates the number of $11 / 12$ and 22 homozygotes in the combined single vessel disease and multiple vessel disease cohort $(L+S)$.

ratios (OR) with 95\% confidence intervals (CI) were also calculated. To summarise results over the Leicester and Sheffield cohorts, MantelHaenszel analyses were performed. Nominal evidence for association was assumed at a probability value of $p=0.05$, with a corrected value of $p=0.013$ being used for studywide significance, which takes into account the multiple testing. Here we have corrected for the four loci tested. However, owing to linkage disequilibrium between these loci this correction is likely to be conservative. IL-1RN (VNTR) was collapsed and analysed as a biallelic marker, as very few genotypes were recorded with the rarer alleles. Neither of the cohorts studied was significantly different from the Hardy Weinberg equilibrium for any of the polymorphism.

Demographic data are expressed as per cent with actual counts in parentheses. These variables were compared by the $\chi^{2}$ test. blood using standard methods and polymerase chain reaction (PCR) for variants within IL-1 related loci, performed as described previously $^{15}$ or using an automated Taqman FRET based system. The less common interleukin receptor antagonist gene variant (IL-1RN allele 2) is referred to as IL- $1 \mathrm{RN}^{\star} 2$.

STATISTICS

Data from Sheffield and Leicester are presented both separately and combined to reveal the trends in each dataset. Differences in genotype distribution were assessed by $\chi^{2}$ analysis of the relevant $2 \times 2$ contingency table. Odds

\section{Results}

DEMOGRAPHICS

The Sheffield and Leicester combined cohorts were well matched for baseline clinical features (table 1).

GENETIC ANALYSIS

The Mantel-Haenzsel results summarised over the Leicester and Sheffield cohorts showed no significant differences in genotypic 
Table 2 Carriage of interleukin 1 receptor antagonist gene (IL-1RN) alleles in the Sheffield and Leicester restenosis and non-restenosis cohorts

\begin{tabular}{llllll}
\hline & \multicolumn{2}{l}{ Leicester SVD and $M V D$} & & \multicolumn{2}{l}{ Sheffield SVD } \\
\cline { 2 - 3 } \cline { 5 - 6 } & $11 / 12^{\star}$ & $22 \dagger$ & & $11 / 12^{\star}$ & $22 \dagger$ \\
\hline Restenosis (raw data) & 46 & 3 & & 16 & 0 \\
Non-restenosers (raw data) & 58 & 10 & & 25 & 7 \\
p Value & 0.1691 & & & 0.0384 & \\
Odds ratio (95\% CI) & $2.6(0.7$ to 10.2) & & N/A & \\
MH p value & 0.0196 & & N/A & \\
\hline
\end{tabular}

$\star 11 / 12$, raw numbers of 1,1 and 1,2 alleles at IL-1RN (VNTR). Allele 1 has four repeats and allele 2 has two repeats of 86 base pairs.

$\dagger 22$, raw numbers of 2,2 homozygotes at IL-1RN (VNTR).

$\mathrm{N} / \mathrm{A}$, Odds ratio and $\mathrm{p}$ value not applicable as one of the values in the contingency table is 0 .

MH, Mantel-Haenzsel summary statistic; MVD, multiple vessel disease; SVD, single vessel disease.

Table 3 Homozygosity at IL-1RN*2 illustrates the difference between single vessel disease (SVD) and multiple vessel disease (MVD) in the Leicester and Sheffield cohorts

\begin{tabular}{|c|c|c|c|c|}
\hline & \multicolumn{4}{|c|}{ Alleles $I L-1 R N$} \\
\hline & \multicolumn{2}{|l|}{$S V D$} & \multicolumn{2}{|l|}{$M V D$} \\
\hline & $11 / 12^{\star}$ & $22 \dagger$ & $11 / 12^{\star}$ & $22 \dagger$ \\
\hline \multicolumn{5}{|l|}{ Leicester } \\
\hline Restenosis & 24 & 1 & & 2 \\
\hline Non-restenosis & 35 & 7 & 23 & 3 \\
\hline p Value & \multirow{2}{*}{\multicolumn{2}{|c|}{$\begin{array}{l}0.1573 \\
4.8(0.6 \text { to } 41.6)\end{array}$}} & \multirow{2}{*}{\multicolumn{2}{|c|}{$\begin{array}{l}0.7669 \\
1.4(0.1 \text { to } 4.6)\end{array}$}} \\
\hline Odds ratio $(95 \% \mathrm{CI})$ & & & & \\
\hline \multicolumn{5}{|l|}{ Sheffield } \\
\hline Restenosis & 16 & 0 & N/A & N/A \\
\hline Non-restenosis & 25 & 7 & & \\
\hline $\mathrm{p}$ Value & \multicolumn{4}{|l|}{0.0384} \\
\hline Odds ratio $(95 \% \mathrm{CI})$ & \multicolumn{4}{|l|}{ N/A } \\
\hline $\mathrm{MH} \mathrm{p}$ value & \multicolumn{4}{|l|}{0.0131} \\
\hline
\end{tabular}

distributions at the IL-1A (-889), IL-1B $(+3954)$, and IL-1B (-511) loci between restenosers and non-restenosers (data not shown).

IL- $1 \mathrm{RN}^{\star} 2$ was increased in the nonrestenoser group compared with the restenosers (34\% v 23\%, respectively ( $\mathrm{L}+\mathrm{S})$, fig 1$)$. IL-1RN*2 homozygosity was significantly increased in the non-restenoser population compared with the restenosers (MantelHaenszel test: $\mathrm{p}=0.0196(\mathrm{~L}+\mathrm{S})$, table 2; $\mathrm{OR}_{(\mathrm{L}+\mathrm{S})}=4.2,95 \% \mathrm{CI} 1.2$ to 15.1$)$. This effect seemed to be restricted to the single vessel disease subpopulation only. The analysis for multivessel disease alone was non-significant $(\mathrm{p}=0.7669(\mathrm{~L}) ; \mathrm{OR}=1.4,95 \% \mathrm{CI} 0.1$ to 4.6 ; table 3$)$; in addition, the result using only single vessel disease patients across both populations remained significant (single vessel disease only, Mantel-Haenszel test: $\mathrm{p}=0.0131$ $(\mathrm{L}+\mathrm{S})$, table $3 ; \mathrm{OR}_{(\mathrm{L}+\mathrm{S})}=9.3,95 \%$ CI 1.2 to 73.8).

A further indication that the results are more specifically applicable to single vessel disease is that when carriage of IL- $1 \mathrm{RN}{ }^{\star} 2$ was compared between the single vessel and multiple vessel disease groups in the Leicester cohort, a significant difference was found in nonrestenosers $(p=0.0342)$. This result was confirmed when the Sheffield non-restenoser single vessel disease data were added $(\mathrm{p}=0.0314)$.

\section{Discussion}

These data suggest a genetic susceptibility to restenosis mediated by polymorphism within IL-1 related loci. Specifically, the data presented here indicate that IL- $1 \mathrm{RN}^{\star} 2$ is associated with a lower restenosis rate in patients with single vessel disease. This supports previous data indicating that there are distinct populations with different propensities to restenosis, and that the process is at least to some extent patient related rather than lesion dependent. ${ }^{23}$ Our previous data, ${ }^{15}$ showing that IL- $1 R^{\star} 2$ is associated with single vessel disease on the basis of angiography, led us to speculate that there may be a true genetic distinction between single vessel disease and multiple vessel disease. If so, this might indicate that the IL-1RN` 2 genotype could either lead more rapidly to single vessel disease or be protective against progression to multiple vessel disease. The data presented here add to this.

As restenosis is a biological phenomenon characterised by an early inflammatory response, these new data suggest that IL- $1 \mathrm{RN}^{\star} 2$ may modulate the arterial wall response to injury in such a way as to reduce the likelihood of restenosis. It is known that coronary angioplasty evokes monocyte activation ${ }^{16}$ and that the ability of monocytes in vitro to produce IL-1 correlates with lumen renarrowing after PTCA. ${ }^{17}$ While there are many potential mechanisms whereby this could occur, a protective or beneficial effect of IL- $1 R^{\star} \star 2$ upon vessel wall healing in response to injury is suggested. This might also support the hypothesis formed in our earlier study that IL- $1 \mathrm{RN}^{\star} 2$ slows progression towards multiple vessel disease. ${ }^{15}$

The mechanism whereby IL- $1 \mathrm{RN}^{\star} 2$ modulates the vessel wall response to injury is unclear. This polymorphism has functional correlates but these appear highly cell type specific. ${ }^{18}{ }^{19}$ In monocytes, IL- $1 \mathrm{RN}{ }^{\star} 2$ has been associated with increased IL-1 receptor antagonist (IL-1ra) production under basal and stimulated conditions, ${ }^{18}{ }^{20}$ but not in all populations $^{19}$ or when measured as IL-1RN mRNA. ${ }^{21}$ In contrast, within cells of the columnar epithelium in inflammatory bowel disease $^{22}$ and in endothelial cells ${ }^{23}$ IL- $1 R^{\star}{ }^{\star} 2$ is associated with reduced production of IL-1 ra. The isoform of IL-1ra produced by monocytes is primarily the secreted isoform, whereas that produced by endothelial cells is the larger intracellular form. ${ }^{23}$ The precise function of the intracellular form of IL-1 ra is not known at present.

As the inflammatory influx seen following experimental PTCA in pigs is highly neutrophilic and IL-1B staining abundantpredominantly in the luminal endothelium even into the later phase of healing ${ }^{14}$-we speculate that the relatively proinflammatory endothelial cell phenotype created by the IL-1RN`2 genotype may be important in PTCA. This suggests that modifying the inflammatory response at the time of injury may indeed be beneficial, acting to limit the healing response that leads to luminal renarrowing. 
The IL-1RN VNTR polymorphism is known to be in linkage disequilibrium with other IL-1 related loci, ${ }^{24}$ and although there are some weakly consistent trends for IL-1A $(+4845)$ and IL-1B (+3954), there are no other significant associations with restenosis or nonrestenosis for the other IL-1 polymorphism within the cluster. Hence a specific complex haplotype is not supported by these data. However, linkage disequilibrium between this polymorphism and other unidentified gene polymorphism cannot be excluded.

Owing to subdivision of the data, this study has small sample sizes for many of the analyses performed. This reduces power and to some extent the reliability and confidence in these findings. However, the results here are strengthened by the fact that two separate cohorts were collected, and that very similar directional trends were found in both populations. It was consistently found that summarising over the two cohorts strengthened evidence for association, which further illustrates the concordance. It is, of course, possible that spurious results could have arisen owing to genetic admixture within the cohorts, but again the consistency between the two populations argues against this.

We favour the interpretation that polymorphic variation within the IL-1 related loci has an important impact on arterial disease. Our original published work ${ }^{15}$ showed an association with single vessel coronary disease in two independent populations (Sheffield and London). The study reported here shows association with a different clinical phenotype in a population predominantly from Leicester. Other investigators have found an association between IL$1 \mathrm{RN}+2018$ - a single nucleotide polymorphism (SNP) in linkage disequilibrium with IL$1 \mathrm{RN}^{\star} 2$ - and carotid intimal/medial changes in African Americans. ${ }^{25}$ Recently published data have also indicated that IL-1RN+2018 is associated with a lower incidence of restenosis after coronary stenting, particularly in younger patients. ${ }^{26}$ These studies all argue strongly that polymorphism within IL-1 related loci does have an impact on the pathogenesis of atherosclerotic lesions, although the mechanism remains to be elucidated.

The biological control of IL-1 is complex. ${ }^{27}$ IL-1 actions are inhibited by a non-signalling receptor IL-1RII in membrane bound or soluble form and also by IL-1 $\mathrm{ra},{ }^{28}$ which binds without agonist activity to the signalling receptor IL$1 \mathrm{RI}{ }^{29}$ IL-1ra is an acute phase protein and is induced by cytokines and bacterial products. ${ }^{30}$ Concentrations of IL-1 and IL-1 ra in vivo vary in parallel, suggesting a coordinated pattern of regulation. ${ }^{30}$ IL-1ra is detected in the endothelium of diseased coronary arteries, ${ }^{23}$ it inhibits fatty streak formation in the apolipoprotein $\mathrm{E}$ deficient mouse, ${ }^{31}$ and its deletion using knockout technology results in lesions at vessel stress sites and aneurysm formation. ${ }^{32}$ These data strongly implicate IL-1ra in the control of inflammation in the arterial wall.
CONCLUSION

The results reported here suggest an important association between IL- $1 \mathrm{RN}^{\star} 2$ and protection from restenosis in individuals with single vessel disease. They also might suggest that inflammation might be a positive influence rather than wholly negative after arterial injury. Validation studies in larger study groups and a reappraisal of the complex injury repair mechanisms employed by the arterial wall are indicated.

This study was supported by the British Heart Foundation. We are grateful for the assistance of the division of molecular and genetic medicine, University of Sheffield, for automated genotyping.

1 Lau SK, Faxon DP. Restenosis. In: Sigwart U, Bertrand M, Serruys PW, eds. Handbook of cardiovascular interventions. London: Churchill Livingstone, 1996:527-40.

2 Lehmann KG, Melkert R, Serruys PW. Contributions of frequency distribution analysis to the understanding of coronary restenosis: a reappraisal of the gaussian curve. Circulation 1996;93:1123-32.

3 Weintraub WS, Brown CL, Liberman HA, et al. Effect of restenosis at one previously dilated coronary site on the probability of restenosis at another previously dilated probability of restenosis at another previo

4 Samani NJ, Martin DS, Brack M, et al. Insertion deletion polymorphism in the ACE gene and risk of restenosis after polymorphism in the ACE gene and risk of reste
coronary angioplasty. Lancet 1995;345:1013-16.

5 Ohishi M, Fujii K, Minamino T, et al. A potent genetic risk factor for restenosis. Nat Med 1993;5:324-5

6 Kaski JC, Zhang YF, Calvino R, et al Angiotensinconverting enzyme insertion deletion polymorphism and restenosis after coronary angioplasty in unstable angina pectoris. Am 7 Cardiol 1996;77:875-7.

7 Rachhaus M, Francis DP, Schmidt H, et al. Not the ACE polymorphism, but the E-selectin gene polymorphism helps to predict restenosis after coronary angioplasty [abstract]. Circulation 1998;17:2070.

8 Abbate R, Marucci R, Camacho-Venegas O, et al. Role of platelet glycoprotein PL A1/A2 polymorphism in restenosis after percutaneous transluminal coronary angioplasty. $\mathrm{Am}$ f Cardiol 1998;82:524-5.

9 Mamotte CDS, Bockxmeer FM, Taylor RR. P1 A1/A2 polymorphism of glycoprotein III1 and risk of coronary artery disease and restenosis following coronary angioplasty. Am f Cardiol 1998;82:13-16.

10 Samani N, Martin DS, Brack M, et al. Apoplipoprotein E polymorphism does not predict risk of restenosis after coronary angioplasty. Atherosclerosis 1996;125:209-16.

11 Jukema JW, deMaat MP, Zwinderman AH, et al. Risk of clinical restenosis after PTCA is influenced by a common functional variant in the human stromelysin promoter [abstract]. Circulation 1997;96:593.

12 Volzke H, Hertwig S, Rettig R, et al. The angiotensinogen gene $235 \mathrm{~T}$ variant is associated with an increased risk of restenosis after percutaneous transluminal coronary angioplasty. Clin Sci 2000;99:19-25.

13 Ross R. Atherosclerosis - an inflammatory disease. N Engl f Med1999;340:115-26.

14 Chamberlain J, Gunn J, Francis SE, et al. Temporal and spatial distribution of IL-1 $\beta$ in balloon injured coronary arteries. Cardiovasc Res 1999;44:156-65.

15 Francis SE, Camp NJ, Dewberry RM, et al. Interleukin-1 receptor antagonist gene polymorphism and coronary artery disease. Circulation 1999;99:861-6.

16 Serrano C, Ramires JA, Venturinelli M, et al. Coronary angioplasty results in leukocyte and platelet activation with adhesion molecule expression: evidence of inflammatory responses in coronary angioplasty. $\mathcal{F} \mathrm{Am}$ Coll Cardiol 1997; 29:1276-83

17 Pietersma A, Kofflard M, de Wit LE, et al. Late lumen loss after coronary angioplasty is associated with the activation status of circulating phagocytes before treatment. Circulastatus of circulating pha

18 Danis VA, Millington M, Hyland VJ, et al. Cytokine production by normal human monocytes: inter-subject variation and relationship to an IL-1 receptor antagonist (IL-1ra)

19 Mandrup-Poulsen T, Pociot F, Molvig J, et al. Monokine antagonism is reduced in patients with IDDM. Diabetes 1994;43:1242-7.

20 Wilkinson RJ, Patel P, Llewelyn M, et al. Influence of polymorphism in the genes for the interleukin (IL)-1 receptor antagonist and IL-1 $\beta$ on tuberculosis. F Exp Med 1999;189: 1863-73.

21 Clay FE, Tarlow JK, Cork MJ, et al. Novel interleukin-1 receptor antagonist exon polymorphisms and their use in
allele-specific mRNA assessment. Hum Genet 1996;97: 723-6.

22 Carter MJ, Jones S, di Giovine FS, et al. Allele 2 of IL-1RN gene polymorphism is associated with reduced expression of IL-1 ra in ulcerative colitis [abstract]. Gastroenterology 1998;114:3882. 
23 Dewberry RM, Holden $\mathrm{H}$, Crossman DC, et al. Interleukin-1 receptor antagonist (IL-1ra) in human Interleukin-1 receptor antagonist (IL-1ra) in human Thromb Vasc Biol 2000;20:2394-400.

24 Cox A, Camp NJ, Nicklin MJH, et al. An analysis of linkage disequilibrium in the interleukin-1 gene cluster, using a novel grouping method for multiallelic markers. Am f Hum Genet 1998;62:1180-8.

25 Pankow JS, Beck JD, Offenbacher S, et al. Association of interleukin-1 gene variants and carotid arterial wall thickness: the ARIC study [abstract]. Atherosclerosis 1999;144:124.

26 Kastrati A, Koch W, Berger PB, et al. Protective role against restenosis from an interleukin-1 receptor antagonist gene polymorphism in patients treated with coronary stenting. $\mathcal{F}$ Am Coll Cardiol 2000;36:2168-73.

27 Dinarello CA. Interleukin-1 and interleukin-1 antagonism. Blood 1991;77:1627-32.
28 Symons JA, Eastgate JA, Duff GW. Purification and characterisation of a novel soluble receptor for interleukin-1. F Exp Med 1991;177:557-60

29 Symons JA, Young PR, Duff GW. Soluble type II interleukin-1 receptor binds and blocks the processing of IL-1B precursors and loses affinity for IL-1 receptor antagonist. Proc Natl Acad Sci USA 1995;92:1714-18.

30 Arend WP. Interleukin-1 receptor antagonist. Adv Immunol 1993;54:167-227.

31 Elhage R, Maret A, Pieraggi MT, et al. Differential effects of interleukin-1 receptor antagonist and tumour necrosis binding protein on fatty streak formation in apoE-deficient mice. Circulation 1998;97:242-4.

32 Nicklin $\mathrm{MJH}$, Hughes DE, Barton JL, et al. Arterial inflammation in mice lacking the interleukin-1 receptor antagonist gene. F Exp Med 2000;191:303-12.

\section{IMAGES IN CARDIOLOGY}

\section{Subarachnoid haemorrhage presenting as acute myocardial infarction with electromechanical dissociation arrest}

A 58 year old man was admitted to his local emergency department in electromechanical dissociation (EMD), having collapsed at home. Cardiac output soon returned
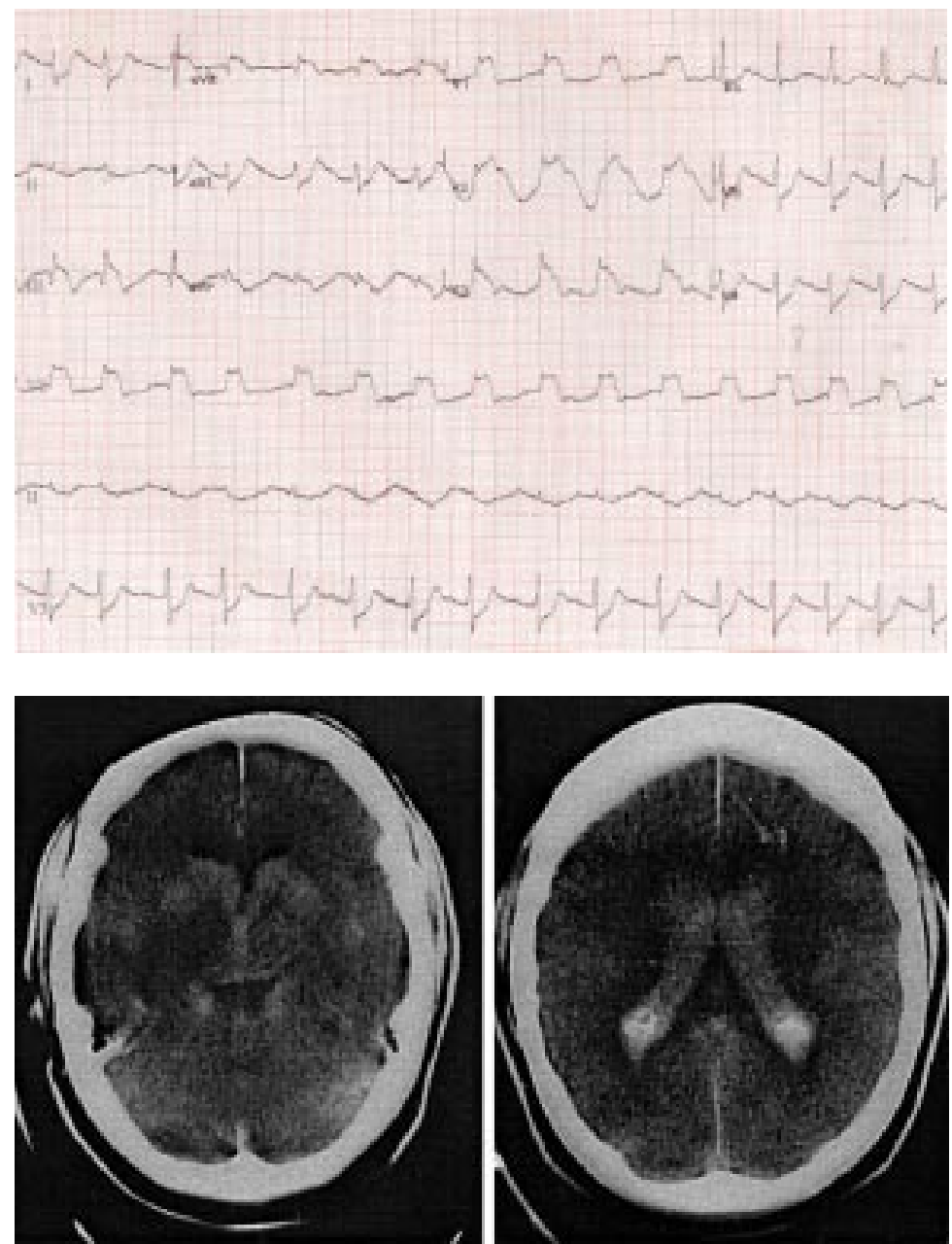

following an adrenaline bolus. His pupils were normal size and reacting, there was no neck stiffness, and he moved his right leg spontaneously. A diagnosis of acute anterior myocardial infarction with EMD arrest was made with ECG evidence of extensive 3-4 mm ST elevation in leads V1-V3, lateral ST depression, and inferior $\mathrm{T}$ wave inversion (top left). He was sedated and paralysed and transferred to a nearby tertiary centre for primary angioplasty rather than thrombolysis in view of a large groin haematoma and recent arrest. Coronary angiography, however, was entirely normal, despite persistent ECG abnormalities. Ventriculography showed a mildly hypokinetic anterior wall. A computed tomographic (CT) scan of the patient's head was arranged to look for an alternative diagnosis. This showed evidence of an extensive subarachnoid haemorrhage with blood throughout the ventricular system and basal cisterns (bottom left). Despite inotropic support, he gradually deteriorated and died 16 hours after admission.

ECG abnormalities occur commonly in subarachnoid haemorrhage, probably caused by subendocardial ischaemia following excessive noradrenaline (norepinephrine) release. Echocardiography is often unhelpful in ruling out an acute coronary syndrome, because of a high prevalence of associated regional wall motion abnormalities in the territory of the ECG changes, as in this case. Patients who present to hospital with ECG evidence of acute myocardial infarction but reduced level of consciousness should undergo an urgent CT head scan to rule out subarachnoid haemorrhage, certainly before thrombolysis is considered.

GAVIN I W GALASKO MIKE DUBOWITZ ROXY SENIOR nphcardiology@netscapeonline.co.uk 